\title{
Common Cesàro hypercyclic vectors
}

\author{
by \\ George Costakis (Heraklion)
}

\begin{abstract}
In this work, which can be seen as a continuation of a paper by Hadjiloucas and the author [Studia Math. 175 (2006)], we establish the existence of common Cesàro hypercyclic vectors for the following classes of operators: (i) multiples of the backward shift, (ii) translation operators and (iii) weighted differential operators. In order to do so, we first prove a version of Ansari's theorem for operators that are hypercyclic and Cesàro hypercyclic simultaneously; then our argument essentially relies on Baire's category theorem. In addition, the minimality of the irrational rotation, Runge's approximation theorem and a common hypercyclicity-universality criterion established by Sambarino and the author [Adv. Math. 182 (2004)], play an important role in the proofs.
\end{abstract}

1. Introduction. We start by recalling the notion of hypercyclicity. Let $X$ be a topological vector space. A linear operator $T: X \rightarrow X$ is called hypercyclic if there exists a vector $x \in X$ such that the sequence $\left\{x, T x, T^{2} x, \ldots\right\}$ is dense in $X$; the vector $x$ is then called hypercyclic for $T$. We will denote by $\mathrm{HC}(T)$ the set of hypercyclic vectors for the operator $T$. In the literature there are many examples of hypercyclic operators such as the translation operator $T_{\alpha} f(z)=f(z+\alpha)$ acting on the space of entire functions $H(\mathbb{C})$, the differentiation operator $D: H(\mathbb{C}) \rightarrow H(\mathbb{C})$, certain unilateral and bilateral weighted shifts, and composition operators on certain function spaces (see the survey article [26]). For more recent results on hypercyclicity we refer to the books [11], [30]; see also the survey articles [13], 24], [27], [28], [37].

Throughout the present paper the symbol $\mathbb{N}$ stands, as usual, for the set of positive integers. For the rest of the introduction $X$ will be a complex separable Banach space, unless otherwise stated. Relatively recently, LeónSaavedra [33] introduced the notion of Cesàro hypercyclicity. Following his terminology, we denote by $M_{n}(T)$ the arithmetic means of an operator $T$ :

2010 Mathematics Subject Classification: Primary 47A16.

Key words and phrases: hypercyclic operators, Cesàro hypercyclicity, residual set, somewhere dense orbit, irrational rotation, Runge's approximation theorem. 
$X \rightarrow X$, that is,

$$
M_{n}(T)=\frac{I+T+\cdots+T^{n-1}}{n}, \quad n=1,2, \ldots
$$

The operator $T$ will be called Cesàro hypercyclic if there is a vector $x$ whose Cesàro orbit under $T,\left\{M_{n}(T) x: n=1,2, \ldots\right\}$, is dense in $X$. Recent studies on Cesàro hypercyclic operators and Cesàro orbits can be found in [20], [33], 35]. Let us also mention that León-Saavedra [33] showed that hypercyclicity does not imply Cesàro hypercyclicity or vice versa.

In [33] it is shown that an operator $T$ is Cesàro hypercyclic if and only if there exists a vector $y$ such that $\left\{n^{-1} T^{n} y: n=1,2, \ldots\right\}$ is dense. We call the vector $y$ Cesàro hypercyclic with respect to the sequence $\left\{n^{-1} T^{n}\right\}$.

We ask the following.

QUESTION 1.1. Is it true that if for some vector $y$ the orbit $\left\{n^{-1} T^{n} y\right.$ : $n=1,2, \ldots\}$ is somewhere dense, then it is dense in $X$ ?

Of course, we can ask the above question by replacing the orbit

$$
\left\{n^{-1} T^{n} y: n=1,2, \ldots\right\}
$$

with the set

$$
\left\{z \in X: \exists n_{k} \rightarrow+\infty, n_{k}^{-1} T^{n_{k}} y \rightarrow z\right\},
$$

since it is not difficult to check that an operator $T$ is Cesàro hypercyclic if and only if there exists a vector $y$ such that the set $\left\{z \in X: \exists n_{k} \rightarrow \infty\right.$, $\left.n_{k}^{-1} T^{n_{k}} y \rightarrow z\right\}$ is dense in $X$. Question 1.1 is inspired by the next two problems:

(i) a corresponding question asked by A. Peris [38] in the setting of hypercyclic operators,

(ii) a problem on the existence of common Cesàro hypercyclic vectors which we shall discuss later.

P. S. Bourdon and N. S. Feldman, in a very nice paper, answered the question raised by Peris [38, proving that: if the orbit $\operatorname{Orb}(T, x)=\left\{T^{n} x\right.$ : $n=0,1,2, \ldots\}$ is somewhere dense then it is everywhere dense in $X$ (see [14]). We now state the following theorem, established in [20], which will be of use to us.

TheOREM 1.2. Let $X$ be a complex separable Banach space and let $T: X \rightarrow X$ be a bounded linear operator. The operator $T$ is hypercyclic and Cesàro hypercyclic if and only if there exist vectors $x$ and $y$ such that the sets $\operatorname{Orb}(T, x)=\left\{T^{n} x: n=0,1,2, \ldots\right\}$ and $F=\left\{z \in X: \exists n_{k} \rightarrow+\infty\right.$, $\left.n_{k}^{-1} T^{n_{k}} y \rightarrow z\right\}$ are somewhere dense. In fact, if $\operatorname{Orb}(T, x)$ and $F$ are somewhere dense in $X$ then both are dense in $X$. 
Using some consequences of Theorem 1.2 (see Section 2), our purpose is to prove the existence of common Cesàro hypercyclic vectors for several classes of operators.

As observed by León-Saavedra the problem of Cesàro hypercyclicity can be seen as the problem on supercyclicity with respect to the sequence $\left\{n^{-1}\right\}$, or as problem of hypercyclicity for a specific sequence of operators (not just the sequence coming from the orbit $\left\{T^{n} x\right\}$ ). To be more precise, let us give the following well known more general definition of hypercyclicity for a sequence of operators (we refer to [26]).

Definition. Let $X$ be a topological vector space (we mainly use as a model space a complex separable Banach space, or the space of entire functions $H(\mathbb{C})$ ). Let $T_{n}: X \rightarrow X$ be a sequence of continuous linear operators. A vector $x \in X$ is called hypercyclic for the sequence of operators $\left\{T_{n}\right\}$ if the sequence $\left\{T_{1} x, T_{2} x, \ldots\right\}$ is dense in $X$. The set of hypercyclic vectors for $\left\{T_{n}\right\}$ will be denoted by $\operatorname{HC}\left(\left\{T_{n}\right\}\right)$.

Using this terminology, we can say that a vector $x$ is Cesàro hypercyclic for an operator $T: X \rightarrow X$ with respect to the sequence $\left\{n^{-1}\right\}$ if $x \in$ $\mathrm{HC}\left(\left\{n^{-1} T^{n}\right\}\right)$. From now on, by the Cesàro orbit of a vector $x$ under $T$ we mean the sequence $\left\{n^{-1} T^{n} x: n=1,2, \ldots\right\}$.

Let us also mention that under some natural assumptions, if the set of hypercyclic vectors for a sequence of operators is non-empty then it is necessarily residual, i.e. it contains a countable intersection of open and dense sets (see for example [26]).

At this point we mention a few facts concerning the minimality of the irrational rotation which will be used frequently throughout this paper. For a real number $x$ the symbol $[x]$ denotes the integer part of $x$. The original and well known statement, known as the minimality of the irrational rotation, says that for every $\theta$ irrational the sequence $\left\{e^{2 \pi i n \theta}: n=0,1,2, \ldots\right\}$ is dense in $\mathbb{T}=\{z \in \mathbb{C}:|z|=1\}$ (see for example [31]). However, we will use the following stronger version of the minimality of the irrational rotation (see [23] for a relevant discussion).

Proposition 1.3. Let $\theta$ be an irrational number. Then for every $\epsilon>0$ there exist a strictly increasing sequence $\left\{n_{k}\right\}$ of positive integers and a positive integer $m$ such that

$$
0 \leq n_{k} \theta-\left[n_{k} \theta\right]<\epsilon \quad \text { for every } k=1,2, \ldots
$$

and

$$
\sup _{k}\left|n_{k+1}-n_{k}\right|<m,
$$

i.e., the sequence $\left\{n_{k}\right\}$ has bounded gaps. 
Let us now describe the main results of the present paper. Applying the results from Section 2 and refining some technics from [22] we shall prove the following theorems.

THEOREM 1.4. Let $T$ be the unilateral backward shift operator acting on $l^{2}(\mathbb{N})$. Then the set $\bigcap_{|\lambda|>1} \operatorname{HC}\left(\left\{n^{-1}(\lambda T)^{n}\right\}\right)$ is residual in $l^{2}(\mathbb{N})$.

Hence the set of common Cesàro hypercyclic vectors for multiples of backward shift is large in the topological sense, i.e. residual. We note that the above theorem has its analogue in the setting of hypercyclic operators for pure powers of $\lambda T$ (see Theorem 4 in [22]).

In 22] we also established the existence of common hypercyclic vectors for all the translation operators $T_{\alpha}: H(\mathbb{C}) \rightarrow H(\mathbb{C}), T_{\alpha}(f)(z):=f(z+\alpha)$, $\alpha \in \mathbb{C} \backslash\{0\}$. Here we prove the following analogue in the Cesàro hypercyclicity setting.

Theorem 1.5. Consider the translation operator $T_{a}: H(\mathbb{C}) \rightarrow H(\mathbb{C})$, $T_{\alpha}(f)(z):=f(z+\alpha), \alpha \in \mathbb{C} \backslash\{0\}$. Then the set $\bigcap_{\alpha \in \mathbb{C} \backslash\{0\}} \operatorname{HC}\left(\left\{n^{-1} T_{\alpha}^{n}\right\}\right)$ is residual in $H(\mathbb{C})$.

As in 22] the proofs of Theorems 1.4 and 1.5 bear some similarity but they are based on quite different approaches. To prove Theorem 1.4 we will use a common universality-hypercyclicity criterion established in [22], while a basic tool for the proof of Theorem 1.5 is Runge's approximation theorem. Of course in both proofs the version of Ansari's theorem for Cesàro hypercyclic operators, which is proven in Section 2, is used along with the minimality of the irrational rotation (Proposition 1.3).

Continuing our investigation of common hypercyclic vectors, we also establish the next theorem, where two parameters appear. In fact, we are able to replace in Theorem 1.5 the weight $n^{-1}$ by the more general weight $n^{b}$ for any real number $b$, but we restrict the translation property to be valid only for non-zero real $\alpha$.

TheOREM 1.6. Let $T_{\alpha}, \alpha \in \mathbb{C} \backslash\{0\}$, be the translation operator acting on the space $H(\mathbb{C})$ of entire functions. Then the set $\bigcap_{b \in \mathbb{R}} \bigcap_{\alpha \in \mathbb{R} \backslash\{0\}} \operatorname{HC}\left(\left\{n^{b} T_{\alpha}\right\}\right)$ is residual in $H(\mathbb{C})$.

It would be very interesting if one could prove a result similar to Theorem 1.6, but allow $\alpha$ to be any non-zero complex number. After the proof of Theorem 1.6, we will explain why our method does not work in this case. In a recent paper [10], Bayart and Matheron were able to solve this problem.

Furthermore it is possible to deal with the differentiation operator acting on $H(\mathbb{C})$, in order to provide common hypercyclic vectors for certain classes of weighted sequences of differential operators. 
TheOREM 1.7. Let $D: H(\mathbb{C}) \rightarrow H(\mathbb{C})$ be the differentiation operator. Then the set $\bigcap_{b \in \mathbb{R}} \mathrm{HC}\left(\left\{n^{b} D^{n}\right\}\right)$ is residual in $H(\mathbb{C})$.

The proof will be based on a straightforward application of the common universality criterion established in [22].

The paper is organized as follows. In Section 2 we establish, as an application of Theorem 1.2, versions of Herrero's conjecture and Ansari's theorem in the context of operators that are hypercyclic and Cesàro hypercyclic. Sections 3 and 4 contain the proofs of Theorems 1.4 and 1.5 respectively. In Section 5, after establishing a series of theorems similar in spirit to Theorem 1.2 and to Theorem 2.7 (a first version of Ansari's result), we prove Theorem 1.6. In the last section, we give a proof of Theorem 1.7.

Let us also list some papers dealing with the subject of common hypercyclic vectors: [1], 3], [10], [12, [15]-[17], [19], [21], 22], [25], [34, [41]-[43]. We also note that E. Abakumov and G. Gordon [1] were the first to establish the existence of common hypercyclic vectors for an uncountable class of operators, the multiples of the backward shift, answering a question raised by H. Salas [40].

2. Applications of Theorem 1.2. In this section we present two applications of Theorem 1.2. More specifically we shall give versions of Herrero's conjecture [32] and Ansari's theorem [2] in the context of operators which are hypercyclic and Cesàro hypercyclic simultaneously. Shamim Ansari [2], using essentially a connectivity argument, showed the following striking result: if $T$ is hypercyclic, then for every positive integer $n, T^{n}$ is also hypercyclic and furthermore $T$ and $T^{n}$ share the same collection of hypercyclic vectors. Recently two new, illuminating proofs of Ansari's theorem have been published: by Grosse-Erdmann, León-Saavedra and Piqueras-Lerena [29] and by Marano and Salas [36. Recall that a positive answer to Herrero's conjecture, that is, the proof that multi-hypercyclic operators are hypercyclic, was given by the author [18] and independently by A. Peris [38]. Observe that Herrero's conjecture implies Ansari's theorem (see for example [38], [14]), while both Herrero's conjecture and Ansari's theorem are corollaries of Bourdon and Feldman's Theorem 2.4 of [14]. Let us also mention that J. Wengenroth [44] was able to relax the hypothesis that $X$ is a Banach space (or more generally a locally convex space). In fact, he showed that the previously mentioned results are valid in arbitrary topological vector spaces.

To continue with a version of Herrero's conjecture, we state a lemma.

Lemma 2.1. Suppose $y_{1}, \ldots, y_{m}$ are vectors in $X$. The following are equivalent:

(i) The set $\left\{n^{-1} T^{n} y_{j}: j=1, \ldots, m, n=1,2, \ldots\right\}$ is dense in $X$.

(ii) $\bigcup_{j=1}^{m}\left\{z \in X: \exists n_{k} \rightarrow+\infty, n_{k}^{-1} T^{n_{k}} y_{j} \rightarrow z\right\}=X$. 


\section{Proof. Trivial.}

We now establish several lemmas in the spirit of 33] León-Saavedra 33 proved that if $T$ is Cesàro hypercyclic, then for every complex number $\lambda$, the operator $T-\lambda$ has dense range. In a similar way we can prove

Lemma 2.2. If $T$ is multi-Cesàro hypercyclic, that is, there exist a positive integer $m$ and vectors $y_{1}, \ldots, y_{m}$ in $X$ such that the set $\bigcup_{j=1}^{m}\left\{M_{n}(T) y_{j}\right.$ : $n=1,2, \ldots\}$ is dense in $X$, then for every complex number $\lambda$, the operator $T-\lambda$ has dense range.

Lemma 2.3. Suppose $y_{1}, \ldots, y_{m}$ are vectors in $X$. The following are equivalent:

(i) The set $\left\{n^{-1} T^{n} y_{j}: j=1, \ldots, m, n=1,2, \ldots\right\}$ is dense in $X$.

(ii) The set $\left\{n^{-1}\left(I-T^{n}\right) y_{j}: j=1, \ldots, m, n=1,2, \ldots\right\}$ is dense in $X$.

Proof. The proof relies on the fact that for $y \in X$ the quantity

$$
\left\|n^{-1} T^{n} y-n^{-1}\left(I-T^{n}\right) y\right\|=n^{-1}\|y\|
$$

converges to 0 as $n \rightarrow+\infty$.

Lemma 2.4. Suppose that the set $\bigcup_{j=1}^{m}\left\{M_{n}(T) y_{j}: n=1,2, \ldots\right\}$ is dense in $X$ for some $y_{1}, \ldots, y_{m} \in X$. Then the set $\left\{n^{-1} T^{n} y_{j}: j=1, \ldots, m, n=\right.$ $1,2, \ldots\}$ is dense in $X$.

Proof. By Lemma 2.2 and our hypothesis we deduce that the set

$$
(T-I)\left(\bigcup_{j=1}^{m}\left\{M_{n}(T) y_{j}: n=1,2, \ldots\right\}\right)=\bigcup_{j=1}^{m}\left\{n^{-1}\left(I-T^{n}\right)\left(y_{j}\right): n=1,2, \ldots\right\}
$$

is dense in $X$. Now Lemma 2.3 implies the desired result.

Below we establish an analogue of Herrero's conjecture for operators which are hypercyclic and Cesàro hypercyclic simultaneously.

TheOREM 2.5. If $T$ is multi-hypercyclic and multi-Cesàro hypercyclic, then $T$ is hypercyclic and Cesàro hypercyclic.

Proof. That $T$ is hypercyclic is already known from the work of Bourdon and Feldman [14]. Since $T$ is multi-Cesàro hypercyclic, there are $y_{1}, \ldots, y_{m} \in$ $X$ such that the set $\bigcup_{j=1}^{m}\left\{M_{n}(T) y_{j}: n=1,2, \ldots\right\}$ is dense in $X$. Applying Lemma 2.4 we find that $\left\{n^{-1} T^{n} y_{j}: j=1, \ldots, m, n=1,2, \ldots\right\}$ is dense in $X$. Now Lemma 2.1 implies that

$$
\overline{\bigcup_{j=1}^{m}\left\{z \in X: \exists n_{k} \rightarrow+\infty, n_{k}^{-1} T^{n_{k}} y_{j} \rightarrow z\right\}}=X .
$$

From this we deduce that for some $i \in\{1, \ldots, m\}$ the set

$$
\left\{z \in X: \exists n_{k} \rightarrow+\infty, n_{k}^{-1} T^{n_{k}} y_{i} \rightarrow z\right\}
$$


is somewhere dense. Finally, Theorem 1.2 ensures that $T$ is Cesàro hypercyclic.

We now turn to an analogue of Ansari's theorem, in the context of hypercyclic and Cesàro hypercyclic operators.

Lemma 2.6. Suppose there exists a vector $y \in X$ such that

$$
\left\{z \in X: \exists n_{k} \rightarrow+\infty, n_{k}^{-1} T^{n_{k}} y \rightarrow z\right\}=X .
$$

Then for every $m=1,2, \ldots$,

$$
\bigcup_{\rho=0}^{m-1}\left\{z \in X: \exists n_{k} \rightarrow+\infty, n_{k}^{-1} T^{m n_{k}}\left(T^{\rho} y\right) \rightarrow z\right\}=X .
$$

Proof. Let us first verify that for every $m=1,2, \ldots$,

$$
\begin{aligned}
m\left\{z \in X: \exists n_{k} \rightarrow\right. & \left.+\infty, n_{k}^{-1} T^{n_{k}} y \rightarrow z\right\} \\
& \subset \bigcup_{\rho=0}^{m-1}\left\{z \in X: \exists n_{k} \rightarrow+\infty, n_{k}^{-1} T^{m n_{k}}\left(T^{\rho} y\right) \rightarrow z\right\} .
\end{aligned}
$$

Let $z \in\left\{w \in X: \exists n_{k} \rightarrow+\infty, n_{k}^{-1} T^{n_{k}} y \rightarrow w\right\}$ and fix a positive integer $m$. Since every natural number $N$ can be expressed as $N=m n+\rho$ for some natural number $n$ and $\rho \in\{0,1, \ldots, m-1\}$, there exists a sequence $\left\{n_{k}\right\}$ of positive integers and some $\rho \in\{0,1, \ldots, m-1\}$ such that $n_{k} \rightarrow+\infty$ and $\left(m n_{k}+\rho\right)^{-1} T^{m n_{k}+\rho} y \rightarrow z$. Now it is not difficult to see that $n_{k}^{-1} T^{m n_{k}}\left(T^{\rho} y\right) \rightarrow m z$. Hence

$$
m z \in\left\{z \in X: \exists n_{k} \rightarrow+\infty, n_{k}^{-1} T^{m n_{k}}\left(T^{\rho} y\right) \rightarrow z\right\} .
$$

Since $\left\{z \in X: \exists n_{k} \rightarrow+\infty, n_{k}^{-1} T^{n_{k}} y \rightarrow z\right\}=X$, the desired result follows.

We are ready to prove a version of Ansari's theorem which will be useful in the next sections.

Theorem 2.7. If $T: X \rightarrow X$ is hypercyclic and for some $y \in X$ the orbit $\left\{n^{-1} T^{n} y: n=1,2, \ldots\right\}$ is dense, then for every positive integer $m$ the orbit $\left\{n^{-1} T^{m n} y: n=1,2, \ldots\right\}$ is also dense. Hence, if $T$ is hypercyclic and Cesàro hypercyclic, then for every positive integer $m, T^{m}$ is hypercyclic and Cesàro hypercyclic.

Proof. Fix a positive integer $m$. Lemma 2.6 implies that for some $\rho \in$ $\{0,1, \ldots, m-1\}$ the set $\left\{z \in X: \exists n_{k} \rightarrow+\infty, n_{k}^{-1} T^{m n_{k}}\left(T^{\rho} y\right) \rightarrow z\right\}$ is somewhere dense. Since $T^{m}$ is hypercyclic (by Ansari's theorem), Theorem 1.2 shows that $\left\{z \in X: \exists n_{k} \rightarrow+\infty, n_{k}^{-1} T^{m n_{k}}\left(T^{\rho} y\right) \rightarrow z\right\}=X$. Therefore the orbit $\left\{n^{-1} T^{m n}\left(T^{\rho} y\right)\right\}$ is dense. Because $T$ is a hypercyclic operator, it has dense range. Hence, the set $T^{m-\rho}\left(\left\{n^{-1} T^{m n}\left(T^{\rho} y\right)\right\}\right)=\left\{n^{-1} T^{m(n+1)} y\right\}$ is dense. From that, it is easy to check that the orbit $\left\{n^{-1} T^{m n} y\right\}$ is dense. 
Remark 2.8. Observe that Theorem 2.7 (and Theorem 1.2), in view of the result of Wengenroth [44, holds for any topological vector space as well. In particular it holds for the complete metric space $H(\mathbb{C})$ of entire functions, endowed with the topology of uniform convergence on compact sets. This remark is crucial for the rest of the paper, especially when we deal with hypercyclic operators acting on $H(\mathbb{C})$. In this section we preferred to work on a complex Banach space, because we frequently wanted to refer to León-Saavedra's paper [33].

3. Proof of Theorem 1.4. Let us first state the following common universality criterion, established in [22], which will be used repeatedly throughout this paper.

Theorem 3.1. Let $X$ be a separable F-space (a topological vector space whose topology is induced by a complete invariant metric $\rho$; to simplify notation we write $\|x\|=\rho(x, 0))$. Let $\left\{T_{n, \lambda}: n \in \mathbb{N}, \lambda \in I\right\}$ be a family of operators on $X$ such that for fixed $n$ the map $\lambda \mapsto T_{n, \lambda}$ is continuous. Assume there is a dense set $\left\{x_{j}: j=1,2, \ldots\right\} \subset X$ and a family $\left\{S_{n, \lambda}: n \in \mathbb{N}, \lambda \in I\right\}$ of operators such that $T_{n, \lambda} \circ S_{n, \lambda}=\operatorname{Id}$ and on $X$ :

(1) Given $x_{j}$ and a compact set $K \subset I$ there is a sequence of positive numbers $c_{k}$ such that

(a) $\sum_{k=1}^{\infty} c_{k}<\infty$

(b) $\left\|T_{n+k, \lambda} \circ S_{n, \alpha} x_{j}\right\| \leq c_{k}$ for any $n, k \geq 0$ and $\lambda, \alpha \in K$,

(c) $\left\|T_{n, \lambda} \circ S_{n+k, \alpha} x_{j}\right\| \leq c_{k}$ for any $n, k \geq 0$ and $\lambda, \alpha \in K$ with $\lambda \leq \alpha$.

(2) Given $\epsilon, x_{j}$ and a compact set $K \subset I$, there exists $0<C(\epsilon)<1$ such that for $\lambda, \alpha \in K$,

$$
\text { if } 1 \geq \lambda / \alpha>C(\epsilon)^{1 / n} \quad \text { then }\left\|T_{n, \lambda} \circ S_{n, \alpha} x_{j}-x_{j}\right\|<\epsilon .
$$

Then there exists a residual set $G \subset X$ such that for every $\lambda \in I$,

$$
G \subset \mathrm{HC}\left(\left\{T_{n, \lambda}\right\}\right) .
$$

In the rest of this section the symbol $T$ denotes the backward shift operator acting on the space $l^{2}(\mathbb{N})$ of square summable sequences. The proof of Theorem 1.4 is based on the next two theorems. We follow the scheme developed in [22].

Theorem 3.2. There is a dense $G_{\delta}$ set $G \subset l^{2}(\mathbb{N})$ such that

$$
G \subset \operatorname{HC}\left(\left\{n^{-1}(\lambda T)^{n}\right\}\right) \quad \text { for every real } \lambda>1 .
$$

Proof. We will apply the common universality-hypercyclicity criterion. For every $n \in \mathbb{N}$ define

$$
T_{n, \lambda}=\frac{(\lambda T)^{n}}{n} \quad \text { and } \quad S_{n, \lambda}=n \frac{S^{n}}{\lambda^{n}}
$$


where $S$ is the forward shift defined by

$$
S\left(x_{1}, x_{2}, \ldots\right)=\left(0, x_{1}, x_{2}, \ldots\right) \quad \text { for }\left(x_{1}, x_{2}, \ldots\right) \in l^{2}(\mathbb{N}) .
$$

It is obvious that $T_{n, \lambda} \circ S_{n, \lambda}=\mathrm{Id}$. As a countable dense set in $l^{2}\left(=l^{2}(\mathbb{N})\right)$ take

$$
D=\left\{\left(x_{n}\right) \in l^{2}: x_{n} \in \mathbb{Q}+i \mathbb{Q} \text { and } x_{n}=0 \text { eventually }\right\} .
$$

To check items (1), (2) of Theorem 4.1, fix $x=\left(x_{n}\right) \in D$, a compact interval $\left[\lambda_{1}, \lambda_{2}\right] \subset(1, \infty)$ and $\epsilon>0$. There is $k_{0}$ such that $x_{k}=0$ for every $k \geq k_{0}$. Working as in the proof of Theorem 15 from [22], set

$$
c_{k}=\frac{k+1}{\lambda^{k}}\|x\|, \quad k \geq k_{0},
$$

and choose $C(\epsilon)$ so that

$$
1-\epsilon /\|x\|<C(\epsilon)<1 .
$$

Then it is easy to check that all the hypotheses of Theorem 3.1 are satisfied. This completes the proof.

Theorem 3.3. Suppose $x \in \mathrm{HC}\left(\left\{n^{-1}(\lambda T)^{n}\right\}\right)$ for some $\lambda>1$. Then

$$
x \in \operatorname{HC}\left(\left\{n^{-1}\left(\lambda e^{2 \pi i \theta} T\right)^{n}\right\}\right) \quad \text { for every } \theta \in[0,1] .
$$

Hence, if $x$ is Cesàro hypercyclic for $\lambda T$ then it is also Cesàro hypercyclic for $\lambda e^{2 \pi i \theta} T$ for every $\theta \in[0,1]$.

Proof. Fix $\theta \in[0,1]$. We shall prove that $\left\{n^{-1}\left(\lambda e^{2 \pi i \theta} T\right)^{n} x\right\}$ is dense.

In case $\theta$ is rational, we argue as in the proof of Theorem 16 from [22], provided that we now use the version of Ansari's theorem for hypercyclic and Cesàro hypercyclic operators, Theorem 2.7 (notice that Theorem 2.7 is applicable, since $\lambda T$ is hypercyclic and Cesàro hypercyclic: see [33]).

Now assume that $\theta$ is irrational. Fix $z \in l^{2} \backslash\{0\}$ and $\epsilon>0$.

Let $y=\left\{y_{j}\right\} \in l^{2}$ be such that $y_{j}$ is eventually zero and

$$
\|y-z\|<\frac{\epsilon}{8} \text {. }
$$

Observe that the series

$$
\sum_{j=1}^{\infty}\left\|\frac{S^{j} y}{\lambda^{j}}\right\|
$$

converges. Hence, there exists a positive integer $l$ satisfying

$$
y_{j}=0 \quad \text { for } j \geq l \quad \text { and } \quad \sum_{j=l}^{\infty}\left\|\frac{S^{j} y}{\lambda^{j}}\right\|<\frac{\epsilon}{8} .
$$

By Proposition 1.3 there exist a positive integer $m$ and a sequence of positive integers $n_{1}<n_{2}<\cdots$ such that

$$
0 \leq \frac{n_{k} \theta}{l}-\left[\frac{n_{k} \theta}{l}\right]<\frac{\epsilon}{4 \pi l\|z\|} \quad \text { and } \quad \sup _{k}\left|n_{k+1}-n_{k}\right|<m l \text {. }
$$


Set

$$
w=l\left[y+\frac{S^{l} y}{\lambda^{l}}+\cdots+\frac{S^{(m-1) l} y}{\lambda^{(m-1) l}}\right] .
$$

Let $\epsilon_{1}$ be a positive number such that

$$
\text { if } \quad\|v-u\|<\epsilon_{1} \text { then }\left\|\frac{(\lambda T)^{t}}{l}(v)-\frac{(\lambda T)^{t}}{l}(u)\right\|<\frac{\epsilon}{8} \quad \text { for } 1 \leq t \leq m l .
$$

Applying Theorem 2.7, choose a positive integer $n$ such that

$$
\left\|\frac{(\lambda T)^{n l} x}{n}-w\right\|<\epsilon_{1}
$$

and

$$
\left|1-\frac{n}{n+j}\right|<\frac{\epsilon_{1}}{\epsilon_{1}+\|w\|}, \quad j=0,1, \ldots, m .
$$

It is plain that there exists a positive integer $n_{k}$ which can be written as

$$
n_{k}=n l+j l
$$

for some positive integer $j$ with $0 \leq j \leq m-1$. We now estimate

$$
\begin{aligned}
& \left\|\frac{(\lambda T)^{n_{k}} x}{n_{k}}-z\right\|=\left\|\frac{(\lambda T)^{j l}}{l}\left(\frac{(\lambda T)^{n l} x}{n+j}\right)-z\right\| \\
& \quad \leq\left\|\frac{(\lambda T)^{j l}}{l}\left(\frac{(\lambda T)^{n l} x}{n+j}\right)-\frac{(\lambda T)^{j l}}{l}\left(\frac{(\lambda T)^{n l} x}{n}\right)\right\|+\left\|\frac{(\lambda T)^{j l}}{l}\left(\frac{(\lambda T)^{n l} x}{n}\right)-z\right\| \\
& \quad=: A+B .
\end{aligned}
$$

Because of (3.5), 3.6) we get

$$
\left\|\frac{(\lambda T)^{n l} x}{n}-\frac{(\lambda T)^{n l} x}{n+j}\right\|=\left|1-\frac{n}{n+j}\right|\left\|\frac{(\lambda T)^{n l} x}{n}\right\|<\epsilon_{1}
$$

and using (3.4) we deduce that

$$
A<\epsilon / 8 \text {. }
$$

It remains to estimate $B$. Using (3.1), (3.2) and (3.5) it follows that

$$
\begin{aligned}
B & \leq\left\|\frac{(\lambda T)^{j l}}{l}\left(\frac{(\lambda T)^{n l} x}{n}\right)-\frac{(\lambda T)^{j l}}{l} w\right\|+\left\|\frac{(\lambda T)^{j l}}{l} w-y\right\|+\|y-z\| \\
& <\epsilon / 8+\epsilon / 8+\epsilon / 8=3 \epsilon / 8 .
\end{aligned}
$$

Therefore,

$$
\left\|\frac{(\lambda T)^{n_{k}} x}{n_{k}}-z\right\|<\frac{\epsilon}{2}
$$


and combining this with $(3.3)$ we arrive at

$$
\begin{aligned}
\left\|\frac{\left(\lambda e^{2 \pi i \theta} T\right)^{n_{k}} x}{n_{k}}-z\right\| & =\left\|e^{2 \pi i n_{k} \theta} \frac{(\lambda T)^{n_{k}} x}{n_{k}}-z\right\| \\
& \leq\left\|\frac{(\lambda T)^{n_{k}} x}{n_{k}}-z\right\|+\left\|e^{2 \pi i n_{k} \theta} z-z\right\| \\
& <\frac{\epsilon}{2}+2 \pi\left|n_{k} \theta-l\left[\frac{n_{k} \theta}{l}\right]\right|\|z\|<\frac{\epsilon}{2}+\frac{\epsilon}{2}=\epsilon .
\end{aligned}
$$

This completes the proof of Theorem 3.3.

It is evident that Theorem 1.4 is an immediate consequence of Theorems 3.2 and 3.3 .

4. Proof of Theorem 1.5. As usual $H(\mathbb{C})$ denotes the space of entire functions endowed with the topology of uniform convergence on compact subsets of $\mathbb{C}$. Throughout this section the symbol $T_{\alpha}$, for $\alpha \in \mathbb{C} \backslash\{0\}$, stands for the translation operator $T_{\alpha}: H(\mathbb{C}) \rightarrow H(\mathbb{C})$ defined by

$$
T_{\alpha}(f)(z):=f(z+\alpha) \quad \text { for every } f \in H(\mathbb{C}) .
$$

Following the scheme developed in [22, our Theorem 1.5 consists of two steps. As mentioned in the introduction, in contrast with the proof of Theorem 1.4, the common hypercyclicity criterion is replaced by an appropriate use of Runge's theorem. Of course the version of Ansari's theorem for hypercyclic and Cesàro hypercyclic operators and the minimality of the irrational rotation are important tools in our approach.

Theorem 4.1. There is a dense $G_{\delta}$ set $G \subset H(\mathbb{C})$ such that

$$
G \subset \operatorname{HC}\left(\left\{n^{-1} T_{e^{2 \pi i \theta}}^{n}\right\}\right) \quad \text { for every } \theta \in[0,1] .
$$

Proof. Let $\left\{\phi_{j}: j \geq 1\right\}$ be a countable dense set in $H(\mathbb{C})$. For $s, j, k, m$ in $\mathbb{N}$ define

$$
\begin{aligned}
& E(s, j, k, m)= \\
& \left\{f \in H(\mathbb{C}): \forall \theta \in[0,1] \exists n=n(\theta) \leq m: \sup _{|z| \leq k}\left|\frac{f\left(z+n e^{2 \pi i \theta}\right)}{n}-\phi_{j}(z)\right|<\frac{1}{s}\right\} .
\end{aligned}
$$

It is not difficult to verify that the set $E(s, j, k, m)$ is open in $H(\mathbb{C})$ for all $s, j, k, m \in \mathbb{N}$ : the proof is similar to that of Lemma 9 in [22] and is omitted. Now define

$$
G:=\bigcap_{s} \bigcap_{j} \bigcup_{m} E(j, s, k, m) .
$$

We shall show that $G$ satisfies the conclusion of Theorem 4.1. In view of Baire's category theorem and since each $E(s, j, k, m)$ is open, it suffices to 
prove that for all $s, j, k \in \mathbb{N}$ the set

$$
\bigcup_{m=1}^{\infty} E(s, j, k, m)
$$

is dense in $H(\mathbb{C})$.

Fix $s, j, k \in \mathbb{N}, g \in H(\mathbb{C})$, a compact set $C$ and $\epsilon>0$. We will be done if we prove the existence of an $f \in H(\mathbb{C})$ and a positive integer $m$ satisfying

$$
f \in E(s, j, k, m)
$$

and

$$
\sup _{z \in C}|f(z)-g(z)|<\epsilon .
$$

Without loss of generality assume that $C \subset\{|z| \leq k\}$; moreover $\phi_{j}$ will be simply denoted by $\phi$. Choose $\delta<1 / 2$ such that

$$
\text { if }|z| \leq k \text { and }|z-w|<\delta \text { then }|\phi(z)-\phi(w)|<\frac{1}{2 s} .
$$

Consider a partition $0=\theta_{0}<\theta_{1}<\cdots<\theta_{l}=1$ to be chosen later. Set

$$
t=2 k+1 \quad \text { and } \quad B=\{|z| \leq k+\delta\} .
$$

For $d=0,1, \ldots, l$ define

$$
B_{d}=B+e^{2 \pi i \theta_{d}}(d+1) t .
$$

Observe that the sets $B, B_{0}, B_{1}, \ldots, B_{l}$ are pairwise disjoint. Now define the function $h$ on the compact set $R=B \cup \bigcup_{d=0}^{l} B_{d}$ by

$$
h(z)= \begin{cases}g(z), & z \in B, \\ \phi\left(z-e^{2 \pi i \theta_{d}}(d+1) t\right)(d+1) t, & z \in B_{d}, d=0,1, \ldots, l .\end{cases}
$$

Since $R$ has connected complement, by Runge's theorem (see [39]), there is a polynomial $f$ such that

$$
\sup _{z \in R}|f(z)-h(z)|<\min \left\{\frac{1}{2 s}, \epsilon\right\} .
$$

In the rest of the proof we shall choose $l$ and a partition $0=\theta_{0}<\theta_{1}<\cdots<$ $\theta_{l}=1$ so that $f$ is the desired function. The definition of $h$ and 4.4 imply

$$
\sup _{z \in C}|f(z)-g(z)|<\epsilon,
$$

therefore (4.2) holds. To finish the proof we have to ensure that (4.1) also holds. For that, we shall find $l$ and $0=\theta_{0}<\theta_{1}<\cdots<\theta_{l}=1$ such that for $\theta \in\left[\theta_{d}, \theta_{d+1}\right)$, we get

$$
\sup _{|z| \leq k}\left|\frac{f\left(z+(d+1) t e^{2 \pi i \theta}\right)}{(d+1) t}-\phi(z)\right|<\frac{1}{s} .
$$


Then setting $m=(l+1) t$ we derive 4 .1). Let $\theta \in\left[\theta_{d}, \theta_{d+1}\right)$ and assume for the moment that

$$
\left|e^{2 \pi i \theta_{d+1}}-e^{2 \pi i \theta_{d}}\right|(d+1) t<\delta .
$$

For $|z| \leq k$ we get $z+(d+1) t e^{2 \pi i \theta} \in B_{d}$. Hence, for $|z| \leq k$,

$$
\begin{aligned}
& \left|\frac{f(z+}{\left.(d+1) t e^{2 \pi i \theta}\right)}-\phi(z)\right| \\
& \quad \leq\left|\frac{f\left(z+(d+1) t e^{2 \pi i \theta}\right)}{(d+1) t}-\phi\left(z+(d+1) t e^{2 \pi i \theta}-(d+1) t e^{2 \pi i \theta_{d}}\right)\right| \\
& \quad+\left|\phi\left(z+(d+1) t\left(e^{2 \pi i \theta}-e^{2 \pi i \theta_{d}}\right)\right)-\phi(z)\right| .
\end{aligned}
$$

Now we estimate the right hand side of the above inequality. The definition of $h, 4.4$ and the fact that $(d+1) t>1$ imply

$$
\left|\frac{f\left(z+(d+1) t e^{2 \pi i \theta}\right)}{(d+1) t}-\phi\left(z+(d+1) t e^{2 \pi i \theta}-(d+1) t e^{2 \pi i \theta_{d}}\right)\right|<\frac{1}{2 s} .
$$

Furthermore, (4.3) and (4.6) yield

$$
\left|\phi\left(z+(d+1) t\left(e^{2 \pi i \theta}-e^{2 \pi i \theta_{d}}\right)\right)-\phi(z)\right|<\frac{1}{2 s} .
$$

Hence, 4.5 holds. It only remains to find $l$ and the partition $0=\theta_{0}<\theta_{1}<$ $\cdots<\theta_{l}=1$ so that 4.6 is satisfied. But this can be done exactly as in the proof of Lemma $10 \mathrm{in} \mathrm{[22].} \mathrm{This} \mathrm{completes} \mathrm{the} \mathrm{proof} \mathrm{that} G$ is dense and hence that of Theorem 4.1 .

Theorem 4.2. Let $f \in \mathrm{HC}\left(\left\{n^{-1} T_{e^{2 \pi i \theta}}^{n}\right\}\right)$ for some $\theta$. Then for any positive real number $r$, the function $f$ also belongs to $\operatorname{HC}\left(\left\{n^{-1} T_{r e^{2 \pi i \theta}}^{n}\right\}\right)$.

Proof. It is not difficult to see (for example using a Baire's category argument along with Runge's approximation theorem) that $T_{\alpha}$ is Cesàro hypercyclic for any $\alpha \in \mathbb{C} \backslash\{0\}$, that is, for every $\alpha \in \mathbb{C} \backslash\{0\}$ there is $f \in H(\mathbb{C})$ so that the sequence $\left\{n^{-1} T_{\alpha}^{n} f\right\}$ is dense in $H(\mathbb{C})$. Hence, in our case, the conclusion of Theorem 2.7 (see also Remark 2.8) holds. Suppose $f$ is hypercyclic for the sequence $\left\{n^{-1} T_{e^{2 \pi i \theta}}^{n}\right\}$. Fix $r>0$. We shall prove that $f$ is also hypercyclic for $\left\{n^{-1} T_{r e 2 \pi i \theta}^{n}\right\}$. Fix $g \in H(\mathbb{C})$, a compact set $L \subset \mathbb{C}$ and $\epsilon>0$. We want to prove that there is some positive integer $N$ such that

$$
\sup _{z \in L}\left|\frac{f\left(z+N r e^{2 \pi i \theta}\right)}{N}-g(z)\right|<\epsilon .
$$

If $r=p / q$ is rational, using Theorem 2.7 and the argument in the proof of Theorem 8 in [22], the result easily follows. We concentrate on the case where $r$ is irrational. The proof requires a few steps.

STEP 1: The role of the uniform continuity of $g$ and the minimality of the irrational rotation. 
In this step we define a real number $\delta>0$, a positive integer $k$ and two sequences of positive integers $n_{1}<n_{2}<\cdots$ and $m_{1} \leq m_{2} \leq \cdots$, having certain "approximation properties", by using the uniform continuity of $g$ on $L$ and the minimality of the irrational rotation. So, choose a positive integer $k$ such that

$$
k>2 \sup _{z \in L}|z| .
$$

Let $\delta$ be a positive number such that $\delta<1$ and

$$
\text { if } z \in L \text { and }|z-w|<\delta \text { then }|g(z)-g(w)|<\epsilon / 4
$$

By Proposition 1.3 there exists a sequence of positive integers $n_{1}<n_{2}<\cdots$ such that for every $j=1,2, \ldots$,

$$
\begin{aligned}
0 & \leq \frac{n_{j} r}{k}-\left[\frac{n_{j} r}{k}\right]<\frac{\delta}{k}, \\
\frac{\delta}{n_{j} r} & <\frac{\epsilon}{7 \epsilon+8 \sup _{z \in L}|g(z)|}
\end{aligned}
$$

and

$$
\sup _{j}\left|n_{j+1}-n_{j}\right|<\infty .
$$

By (4.8) and since $\delta<1$ it follows that the integer part of $n_{j} r,\left[n_{j} r\right]$, is a multiple of $k$. Setting $m_{j} k=\left[n_{j} r\right]$, we get

$$
0 \leq n_{j} r-m_{j} k<\delta
$$

and

$$
\sup _{j}\left|m_{j+1}-m_{j}\right|<m
$$

for some positive integer $m$.

STEP 2: The auxiliary function $h$ which we want to approximate on an appropriate set $K$.

Let $d(z, L)$ denote the distance from $z \in \mathbb{C}$ to the compact set $L$. Set

$$
\begin{aligned}
L_{\delta} & =\{z: d(z, L) \leq \delta\}, \quad L_{\delta}^{l}=L_{\delta}+l k e^{2 \pi i \theta}, \quad l=1, \ldots, m-1, \\
K & =L_{\delta} \cup L_{\delta}^{1} \cup \cdots \cup L_{\delta}^{m-1} .
\end{aligned}
$$

Observe that, by the choice of $k$ in Step 1 , the sets $L_{\delta}, L_{\delta}^{1}, \ldots, L_{\delta}^{m-1}$ are pairwise disjoint. We now define the function $h$ on $K$ by

$$
h(z)= \begin{cases}g(z) / r, & z \in L_{\delta}, \\ g\left(z-l k e^{2 \pi i \theta}\right) / r, & z \in L_{\delta}^{l}, l=1, \ldots, m-1 .\end{cases}
$$

STEP 3: We shall prove the following 
Claim 1. There exists a positive integer $j$ such that

$$
\sup _{z \in L}\left|\frac{f\left(z+n_{j} r e^{2 \pi i \theta}\right)}{n k}-\frac{g(z)}{r}\right|<\frac{3 \epsilon}{4 r},
$$

where $k$ is the positive integer from Step 1 and $n_{j}$ is the $j$ th term of the sequence $n_{1}<n_{2}<\cdots$ which appears in Step 1 as well.

Proof. By Runge's theorem there exists an entire function $\xi$ such that

$$
\sup _{z \in K}|\xi(z)-h(z)|<\frac{\epsilon}{4 r} .
$$

Since $f$ is hypercyclic for $\left\{n^{-1} T_{e^{2 \pi i \theta}}^{n}\right\}$, Theorem 2.7 shows that the sequence $\left\{n^{-1} T_{e^{2 \pi i \theta}}^{k n} f\right\}$ is dense in $H(\mathbb{C})$. Then the sequence $\left\{k^{-1} n^{-1} T_{e^{2 \pi i \theta}}^{k n} f\right\}$ is also dense in $H(\mathbb{C})$. Hence, we may find a positive integer $n$ satisfying

$$
\sup _{z \in K}\left|\frac{f\left(z+n k e^{2 \pi i \theta}\right)}{n k}-\xi(z)\right|<\frac{\epsilon}{4 r},
$$

and

$$
\left|\frac{n k}{n k+\rho k}-1\right|<\frac{\epsilon}{6 \epsilon+8 \sup _{z \in L}|g(z)|},
$$

for every $\rho=0,1, \ldots, m-1$. Observe that 4.12 ensures the existence of a positive integer $j$ such that

$$
n k \leq m_{j} k \leq n k+(m-1) k .
$$

In addition there is $l, 0 \leq l \leq m-1$, such that

$$
m_{j} k=n k+l k .
$$

If we set $w=\left(n_{j} r-m_{j} k\right) e^{2 \pi i \theta}$, then 4.11) implies that $|w|<\delta$. Using (4.7), 4.13 and 4.14, for $z \in L$ we get

$$
\begin{aligned}
\left|\frac{f\left(z+n_{j} r e^{2 \pi i \theta}\right)}{n k}-\frac{g(z)}{r}\right| \\
=\left|\frac{f\left(z+\left(n_{j} r-m_{j} k\right) e^{2 \pi i \theta}+m_{j} k e^{2 \pi i \theta}\right)}{n k}-\frac{g(z)}{r}\right| \\
\leq\left|\frac{f\left(z+w+(l k+n k) e^{2 \pi i \theta}\right)}{n k}-\xi\left(z+w+l k e^{2 \pi i \theta}\right)\right| \\
\quad+\left|\xi\left(z+w+l k e^{2 \pi i \theta}\right)-\frac{g(z+w)}{r}\right|+\left|\frac{g(z+w)}{r}-\frac{g(z)}{r}\right| \\
<\frac{\epsilon}{4 r}+\frac{\epsilon}{4 r}+\frac{\epsilon}{4 r}=\frac{3 \epsilon}{4 r} .
\end{aligned}
$$

Hence,

$$
\sup _{z \in L}\left|\frac{f\left(z+n_{j} r e^{2 \pi i \theta}\right)}{n k}-\frac{g(z)}{r}\right|<\frac{3 \epsilon}{4 r}
$$


STEP 4: In this last step we shall prove the following claim which completes the proof of the theorem.

Claim 2. For the positive integer $j$ of Step 3 we have

$$
\sup _{z \in L}\left|\frac{f\left(z+n_{j} r e^{2 \pi i \theta}\right)}{n_{j}}-g(z)\right|<\epsilon .
$$

Proof. Using 4.15 and 4.16 we get

$$
\begin{aligned}
\left|\frac{f\left(z+n_{j} r e^{2 \pi i \theta}\right)}{n k+l k}-\frac{f\left(z+n_{j} r e^{2 \pi i \theta}\right)}{n k}\right| \\
\leq\left|1-\frac{n k}{n k+l k}\right|\left|\frac{f\left(z+n_{j} r e^{2 \pi i \theta}\right)}{n k}\right|<\frac{\epsilon}{8 r} .
\end{aligned}
$$

Moreover combining (4.9), 4.16), 4.17) and using the inequality

$$
\left|1-\frac{\left[n_{j} r\right]}{n_{j} r}\right|<\frac{\delta}{n_{j} r}
$$

(recall that $\left.n k+l k=\left[n_{j} r\right]\right)$, it follows that

$$
\begin{aligned}
\left|\frac{f\left(z+n_{j} r e^{2 \pi i \theta}\right)}{n k+l k}-\frac{f\left(z+n_{j} r e^{2 \pi i \theta}\right)}{n_{j} r}\right| \\
\leq\left|1-\frac{n k+l k}{n_{j} r}\right|\left|\frac{f\left(z+n_{j} r e^{2 \pi i \theta}\right)}{n k+l k}\right|<\frac{\epsilon}{8 r} .
\end{aligned}
$$

Hence, 4.16-4.18 imply

$$
\left|\frac{f\left(z+n_{j} r e^{2 \pi i \theta}\right)}{n_{j} r}-\frac{g(z)}{r}\right|<\frac{\epsilon}{r} .
$$

So we arrive at

$$
\sup _{z \in L}\left|\frac{f\left(z+N r e^{2 \pi i \theta}\right)}{N}-g(z)\right|<\epsilon
$$

for $N=n_{j}$. This completes the proof of Theorem 4.2.

Theorems 4.1 and 4.2 immediately imply Theorem 1.5.

5. Some extensions. In this section we shall describe some extensions of the results presented in previous sections. Our goal is to establish a version of Ansari's theorem for more general sequences of operators than considered before, and then use it to prove the existence of common hypercyclic vectors. We recall that an $F$-space is a topological vector space whose topology is induced by a complete invariant metric $\rho$. To simplify notation we write $\|x\|=\rho(x, 0)$. Let us start with a version of Theorem 1.2. 
TheOREM 5.1. Let $T$ be a hypercyclic operator acting on an F-space $X$. Fix a real number $b$. Suppose there exists $y \in X$ such that the set

$$
F=\left\{z \in X: \exists n_{k} \rightarrow+\infty, n_{k}^{b} T^{n_{k}} y \rightarrow z\right\}
$$

is somewhere dense. Then $F$ is dense in $X$ and since $F$ is closed, we conclude $F=X$.

Proof. The proof is along the lines of the proof of Theorem 1.2 and the required details are left to the reader.

The next lemma is analogous to Lemma 2.6.

Lemma 5.2. Let $T$ be an operator acting on an $F$-space and $b \in \mathbb{R}$. Suppose there exists a vector $y \in X$ such that

$$
\left\{z \in X: \exists n_{k} \rightarrow+\infty, n_{k}^{b} T^{n_{k}} y \rightarrow z\right\}=X .
$$

Then for every $m=1,2, \ldots$,

$$
\bigcup_{\rho=0}^{m-1}\left\{z \in X: \exists n_{k} \rightarrow+\infty, n_{k}^{b} T^{m n_{k}} y \rightarrow z\right\}=X .
$$

Proof. It suffices to check that

$$
\begin{aligned}
m^{-b}\left\{z \in X: \exists n_{k} \rightarrow+\infty\right. & \left., n_{k}^{b} T^{n_{k}} y \rightarrow z\right\} \\
& \subset \bigcup_{\rho=0}^{m-1}\left\{z \in X: \exists n_{k} \rightarrow+\infty, n_{k}^{b} T^{m n_{k}} y \rightarrow z\right\} .
\end{aligned}
$$

The proof is as in the case $b=-1$ (see Lemma 2.6).

We now give a new version of Ansari's result.

TheOREM 5.3. Suppose $T$ is a hypercyclic operator acting on an $F$-space. Fix a real number $b$ and assume that for some $y \in X$ the orbit $\left\{n^{b} T^{n} y\right\}$ is dense in $X$. Then, for every positive integer $m$, the orbit $\left\{n^{b} T^{m n} y\right\}$ is also dense.

Proof. Using Theorem 5.1 and Lemma 5.2, we can argue as in the proof of Theorem 2.7.

The rest of this section is devoted to the proof of Theorem 1.6. The version of Ansari's theorem we established above plays an essential role in our argument. Following the standard procedure (see Sections 3, 4), we next prove two theorems.

TheOREM 5.4. Fix any two real numbers $\lambda_{1}<\lambda_{2}$ and a non-zero real number $\alpha$. There is a dense $G_{\delta}$ set $G \subset H(\mathbb{C})$ such that

$$
G \subset \bigcap_{\lambda \in\left[\lambda_{1}, \lambda_{2}\right]} \operatorname{HC}\left(\left\{n^{\lambda} T_{\alpha}^{n}\right\}\right) .
$$


Proof. Suppose that $\alpha>0$ (the case $\alpha<0$ can be proved in a similar fashion). Without loss of generality we may assume that $\alpha=1$. The proof uses ideas from the proof of Theorem 4.1. Actually, in this case the hard part of the proof, which is to provide a denseness result, is a bit different (although of the same flavor since we are mainly dealing with a one-parameter family of operators) from that of the proof of Theorem 4.1, because of the nature of the partition we are forced to choose. This will become clear later in the proof.

Let $\left\{\phi_{j}: j \geq 1\right\}$ be a countable dense set in $H(\mathbb{C})$. For $s, j, k, m \in \mathbb{N}$ consider the set

$$
\begin{aligned}
& E(m, j, s, n)=\left\{f \in H(\mathbb{C}): \text { for every } \lambda \in\left[\lambda_{1}, \lambda_{2}\right] \text { there is } n=n(\lambda) \leq m\right. \\
& \text { such that } \left.\sup _{|z| \leq k}\left|n^{\lambda} f(z+n)-\phi_{j}(z)\right|<1 / s\right\} .
\end{aligned}
$$

Minor modifications in the proof of Lemma 9 from [22] show that the set $E(s, j, k, m)$ is open in $H(\mathbb{C})$ for all $s, j, k, m \in \mathbb{N}$. It suffices to show that the set

$$
\bigcup_{m=1}^{\infty} E(s, j, k, m)
$$

is dense in $H(\mathbb{C})$ for all $s, j, k \in \mathbb{N}$, because then arguing as in the proof of Theorem 4.1 we obtain Theorem 5.4.

Fix $s, j, k \in \mathbb{N}, g \in H(\mathbb{C})$, a compact set $C$ and $\epsilon>0$. We want to find $f \in H(\mathbb{C})$ and a positive integer $m$ such that

$$
f \in E(s, j, k, m)
$$

and

$$
\sup _{z \in C}|f(z)-g(z)|<\epsilon .
$$

For simplicity $\phi_{j}$ will be denoted by $\phi$. We may also assume $C \subset\{|z| \leq k\}$. Choose a sufficiently small positive number $\delta$ such that

$$
\text { if }|z| \leq k \text { and }|1-r|<\delta \text { then }|1-r||\phi(z)|<\frac{1}{2 s} .
$$

Let $\lambda_{1}=b_{0}<b_{1}<\cdots<b_{l}=\lambda_{2}$ be a partition to be chosen later. We define

$$
\begin{gathered}
t=2 k+1, \quad B=\{|z| \leq k\}, \\
B_{d}=B+(d+1) t, \quad d=0,1, \ldots, l, \quad R=B \cup \bigcup_{d=0}^{l} B_{d}
\end{gathered}
$$

and

$$
h(z)= \begin{cases}g(z), & z \in B, \\ {[(d+1) t]^{-b_{d}} \phi(z-(d+1) t),} & z \in B_{d}, d=0,1, \ldots, l .\end{cases}
$$


Observe that $B, B_{0}, B_{1}, \ldots, B_{l}$ are pairwise disjoint. Since $R$ has connected complement, by Runge's theorem there is a polynomial $f$ so that

$$
\sup _{z \in R}|f(z)-h(z)|<\min \left\{\frac{1}{2 s}, \epsilon\right\} .
$$

Obviously, the definition of $h$ and (5.4) yield

$$
\sup _{z \in C}|f(z)-g(z)|<\epsilon,
$$

hence $(5.2)$ holds. It remains to show that (5.1) also holds. For that we shall choose $l$ and a partition $\lambda_{1}=b_{0}<b_{1}<\cdots<b_{l}=\lambda_{2}$, such that for $b \in\left[b_{d}, b_{d+1}\right)$

$$
\sup _{|z| \leq k}\left|[(d+1) t]^{b} f(z+(d+1) t)-\phi(z)\right|<\frac{1}{s} .
$$

Then, setting $m=(l+1) t$ we obtain (5.1). Observe that

$$
\text { if }|z| \leq k \text { then } z+(d+1) t \in B_{d} .
$$

Thus for every $z$ with $|z| \leq k$ we obtain

$$
\begin{aligned}
& \left|[(d+1) t]^{b} f(z+(d+1) t)-\phi(z)\right| \\
& \leq\left|[(d+1) t]^{b} f(z+(d+1) t)-[(d+1) t]^{b-b_{d}} \phi(z+(d+1) t-(d+1) t)\right| \\
& \quad+\left|[(d+1) t]^{b-b_{d}} \phi(z)-\phi(z)\right| .
\end{aligned}
$$

Let us now bound the right hand side of the above inequality:

$$
\begin{aligned}
& \left|[(d+1) t]^{b} f(z+(d+1) t)-[(d+1) t]^{b-b_{d}} \phi(z+(d+1) t-(d+1) t)\right| \\
& \quad \leq[(d+1) t]^{\lambda_{2}}\left|f(z+(d+1) t)-[(d+1) t]^{-b_{d}} \phi(z+(d+1) t-(d+1) t)\right| .
\end{aligned}
$$

Now (5.4) gives

$$
\left|[(d+1) t]^{b} f(z+(d+1) t)-[(d+1) t]^{b-b_{d}} \phi(z+(d+1) t-(d+1) t)\right| \leq \frac{1}{2 s} .
$$

It remains to bound the term $\left|[(d+1) t]^{b-b_{d}} \phi(z)-\phi(z)\right|$. Observe that this term points out how the partition should be chosen. If

$$
\left|1-[(d+1) t]^{b-b_{d}}\right|<\delta
$$

using (5.3) we arrive at

$$
\left|[(d+1) t]^{b-b_{d}} \phi(z)-\phi(z)\right|<\frac{1}{2 s}
$$

Then (5.7) and (5.9) imply (5.6) and we are done. Therefore, we are left with finding $l$ and the partition $\lambda_{1}=b_{0}<b_{1}<\cdots<b_{l}=\lambda_{2}$, so that (5.8) is satisfied. Since $(d+1) t>1$ and $b \in\left[b_{d}, b_{d+1}\right)$, we notice that

$$
b_{d+1}-b_{d}<\frac{\ln (\delta+1)}{\ln ((d+1) t)}
$$


implies (5.8). If we set

$$
\beta_{d}=b_{d+1}-b_{d}
$$

then 5.10 reads

$$
\beta_{d}<\frac{\ln (\delta+1)}{\ln ((d+1) t)}
$$

It suffices to find $l$ and positive numbers $\beta_{0}, \ldots, \beta_{l-1}$ such that 5.11 holds and

$$
\beta_{0}+\beta_{1}+\cdots+\beta_{l-1}=\lambda_{2}-\lambda_{1} .
$$

Therefore, if we choose $l \geq 1$ so that

$$
\eta=\ln (\delta+1) \sum_{j=0}^{l-1} \frac{1}{\ln ((j+1) t)}>1
$$

and define, for $d=0,1, \ldots, l-1$,

$$
\beta_{d}=\frac{\lambda_{2}-\lambda_{1}}{\eta} \frac{\ln (\delta+1)}{\ln ((d+1) t)},
$$

the relations (5.11), 5.12) are satisfied. This completes the proof of Theorem 5.4.

REMARK 5.5. Theorem 5.4 ensures the existence of an entire function $f$ such that for every $b \in \mathbb{R}$ the sequence $\left\{n^{b} f(z+n)\right\}$ is dense in $H(\mathbb{C})$. However, a stronger and more natural result in this direction would be to establish the existence of a residual set $G$ in $H(\mathbb{C})$ such that for every $f \in G$ the set $\left\{n^{b} f\left(z+e^{2 \pi i \theta} n\right)\right\}$ is dense for every $b \in \mathbb{R}$ and every $\theta \in[0,1]$. Then we shall be able to derive the existence of an entire function $f$ such that the sequence $\left\{n^{b} f(z+n \alpha)\right\}$ is dense for every $b \in \mathbb{R}$ and every $\alpha \in \mathbb{C} \backslash\{0\}$. The difficulty in obtaining a stronger version of Theorem 5.4 is due to the partition. Actually in this case two partitions should be chosen (one for the real numbers $b$ lying on some interval and one for the complex numbers lying on the unit circle). Furthermore, the two partitions are coupled, which creates one more obstacle. To overcome these difficulties it seems that a new idea is needed. Recently Bayart and Matheron, in a very deep paper [10], came up with such an idea. They were able to refine our Theorem 5.4 and prove the existence of a residual set $G$ in $H(\mathbb{C})$ such that for every $f \in G$ the set $\left\{n^{b} f\left(z+e^{2 \pi i \theta} n\right)\right\}$ is dense for every $b \in \mathbb{R}$ and every $\theta \in[0,1]$. For further results concerning common hypercyclic vectors for two-parameter families of operators see [43].

Theorem 5.6. Let $f \in \operatorname{HC}\left(\left\{n^{b} T_{e^{2 \pi i \theta}}^{n}\right\}\right)$ for some $b \in \mathbb{R}$ and $\theta \in[0,1]$. Then $f \in \mathrm{HC}\left(\left\{n^{b} T_{r e^{2 \pi i \theta}}^{n}\right\}\right)$ for any positive real number $r$. 
Proof. It is easy to check that the sequence $\left\{n^{b} T_{\alpha}^{n}\right\}$ is hypercyclic for every fixed $b \in \mathbb{R}$ and $\alpha \in \mathbb{R} \backslash\{0\}$ (and actually for every $\alpha \in \mathbb{C} \backslash\{0\}$ ). Therefore, the conclusion of Theorem 5.3 holds. Making some minor modifications in the proof of Theorem 4.2, such as replacing $n^{-1}$ with $n^{b}$, choosing suitable bounds in (4.10), 4.16) and using Theorem 5.3 instead of Theorem 2.7, we obtain the conclusion. The details are left to the reader.

We are now ready to present the final step of the proof of Theorem 1.6. Assume that $\alpha=1$ in Theorem 5.4. The set $G$ obtained in Theorem 5.4 depends on the choice of the interval $I=\left[\lambda_{1}, \lambda_{2}\right]$, so denote it by $G=G_{I}$. Cover the real line by countably many intervals $I_{n}$, i.e. $\mathbb{R}=\bigcup_{n} I_{n}$, and applying Theorem 5.4, consider the corresponding $G_{I_{n}}$. Then the set $G_{1}=$ $\bigcap_{n} G_{I_{n}}$ is residual in $H(\mathbb{C})$ and $G_{1} \subset \bigcap_{b \in \mathbb{R}} \mathrm{HC}\left(\left\{n^{b} T_{1}^{n}\right\}\right)$. Starting now with $\alpha=-1$ and applying the above procedure, we end up with a set $G_{-1}$ residual in $H(\mathbb{C})$ such that $G_{-1} \subset \bigcap_{b \in \mathbb{R}} \mathrm{HC}\left(\left\{n^{b} T_{-1}^{n}\right\}\right)$. Clearly, $G_{1} \cap G_{-1}$ is residual in $H(\mathbb{C})$ and Theorem 5.6 implies $G_{1} \cap G_{-1} \subset \bigcap_{b \in \mathbb{R}} \bigcap_{\alpha \in \mathbb{R} \backslash\{0\}} \mathrm{HC}\left(\left\{n^{b} T_{\alpha}^{n}\right\}\right)$. This finishes the proof of Theorem 1.6.

6. Proof of Theorem 1.7. The proof of Theorem 1.7 is based on a straightforward application of the common universality-hypercyclicity criterion, Theorem 3.1. Actually we shall first prove two versions of Theorem 1.7 , for $b$ positive and $b$ negative, and then Theorem 1.7 will follow trivially.

TheOREM 6.1. Let $D: H(\mathbb{C}) \rightarrow H(\mathbb{C})$ be the differentiation operator. Then the set $\bigcap_{b>0} \mathrm{HC}\left(\left\{n^{b} D^{n}\right\}\right)$ is residual in $H(\mathbb{C})$.

Proof. Recall that $H(\mathbb{C})$ is a complete metric space with the metric

$$
\rho(f, g)=\sum_{n=1}^{\infty} \frac{1}{2^{n}} \frac{\sup _{|z| \leq n}|f(z)-g(z)|}{1+\sup _{|z| \leq n}|f(z)-g(z)|}
$$

for $f, g \in H(\mathbb{C})$. For convenience we write $\|f\|=\rho(f, 0)$. Define the families of operators $T_{n, \lambda}=n^{\lambda} D^{n}, \lambda>0, S_{n, \lambda}: H(\mathbb{C}) \rightarrow H(\mathbb{C})$, by $S_{n, \lambda}(f)(z)=$ $n^{-\lambda} f^{(-n)}(z)$, where $f^{(-1)}$ is the antiderivative of $f$ whose value at 0 is 0 and $f^{(-n)}=\left(f^{(-(n-1))}\right)^{(-1)}$ for every $n=1,2, \ldots$ Clearly $T_{n, \lambda} \circ S_{n, \lambda}=\mathrm{Id}$ for every positive integer $n$ and every $\lambda>0$. To check properties (1) and (2) of Theorem 3.1 we shall use as a dense set the set of polynomials with coefficients in $\mathbb{Q}+i \mathbb{Q}$. Let $p$ be such a polynomial. Observe that

$$
\begin{aligned}
T_{n+k, \lambda} \circ S_{n, \alpha}(p) & =0 \quad \text { for } k>\operatorname{deg}(p), \\
T_{n, \lambda} \circ S_{n+k, \alpha}(p)(z) & =\frac{n^{\lambda}}{(n+k)^{\alpha}} p^{(-k)}(z) .
\end{aligned}
$$


Therefore, for $\lambda \leq \alpha$ we get

$$
\left\|T_{n, \lambda} \circ S_{n+k, \alpha}(p)\right\| \leq \frac{n^{\lambda}}{(n+k)^{\alpha}}\left\|p^{(-k)}\right\| \leq\left\|p^{(-k)}\right\| .
$$

Defining $c_{k}=\left\|p^{(-k)}\right\|$, it can be shown that $\sum_{k=1}^{\infty} c_{k}<\infty$ (see the proof of Theorem 13 in [22]). Hence, property (1) of Theorem 3.1 is satisfied. It remains to check (2). Let $\epsilon>0, p$ be a polynomial with coefficients in $\mathbb{Q}+i \mathbb{Q}$, $\left[\lambda_{1}, \lambda_{2}\right] \subset(0, \infty)$ be a compact interval and fix $c, 0<c<1$. Then

$$
\left\|T_{n, \lambda} \circ S_{n, \alpha}(p)-p\right\|=\left|\frac{n^{\lambda}}{n^{\alpha}}-1\right|\|p\| .
$$

Since $\lim _{n \rightarrow \infty}\left(1-c^{1 / n}\right) \log n=0$, there is a positive integer $n_{0}$ such that

$$
\left|n^{\lambda_{2}\left(1-c^{1 / n}\right)}-1\right|<\frac{\epsilon}{\|p\|} \quad \text { for } n \geq n_{0} .
$$

Hence, for $n \geq n_{0}$ and $\lambda, \alpha \in\left[\lambda_{1}, \lambda_{2}\right]$ and if $1>\lambda / \alpha>c^{1 / n}$, we deduce that

$$
\left|\frac{n^{\lambda}}{n^{\alpha}}-1\right| \leq\left|\frac{n^{\alpha}}{n^{\lambda}}-1\right|=\left|n^{\alpha(1-\lambda / \alpha)}-1\right|<\frac{\epsilon}{\|p\|} .
$$

Therefore (2) holds for $n \geq n_{0}$ with $1>C(\epsilon)>c$. Now just take $C(\epsilon)$ so that (2) also holds for $n \leq n_{0}$. This completes the proof of Theorem 6.1.

Theorem 6.2. Let $D: H(\mathbb{C}) \rightarrow H(\mathbb{C})$ be the differentiation operator. Then the set $\bigcap_{b<0} \mathrm{HC}\left(\left\{n^{b} D^{n}\right\}\right)$ is residual in $H(\mathbb{C})$.

Proof. The proof is similar to the proof of Theorem 6.1. Indeed, following that proof, it is easy to show that item (1) of Theorem 3.1 is satisfied. It remains to check (2). For that, let $\epsilon>0, p$ be a polynomial with coefficients in $\mathbb{Q}+i \mathbb{Q},\left[\lambda_{1}, \lambda_{2}\right] \subset(-\infty, 0)$ be a compact interval and fix $c, 0<c<1$. Observe that there is a positive integer $n_{0}$ such that

$$
\left|n^{\lambda_{1}\left(1-c^{1 / n}\right)}-1\right|<\frac{\epsilon}{\|p\|} \quad \text { for every } n \geq n_{0} .
$$

Then, arguing as in the proof of Theorem 6.1, one verifies (2).

From Theorems 6.1, 6.2 and the well known fact that the set $\mathrm{HC}(D)$ is residual, Theorem 1.7 follows.

Acknowledgements. I am grateful to the referee. Her/his helpful remarks and illuminating comments considerably improved the presentation of the paper.

\section{References}

[1] E. Abakumov and J. Gordon, Common hypercyclic vectors for multiples of backward shift, J. Funct. Anal. 200 (2003), 494-504. 
[2] S. I. Ansari, Hypercyclic and cyclic vectors, ibid. 128 (1995), 374-383.

[3] R. Aron, J. Bès, F. Léon and A. Peris, Operators with common hypercyclic subspaces, J. Operator Theory 54 (2005), 251-260.

[4] F. Bayart, Common hypercyclic vectors for composition operators, ibid. 52 (2004), 353-370.

[5] —, Common hypercyclic subspaces, Integral Equations Operator Theory 53 (2005), 467-476.

[6] - , Topological and algebraic genericity of divergence and universality, Studia Math. 167 (2005), 161-181.

[7] F. Bayart and S. Grivaux, Hypercyclicity and unimodular point spectrum, J. Funct. Anal. 226 (2005), 281-300.

[8] —, - Frequently hypercyclic operators, Trans. Amer. Math. Soc. 358 (2006), 50835117.

[9] F. Bayart, S. Grivaux and R. Mortini, Common bounded universal functions for composition operators, Illinois J. Math. 52 (2008), 995-1006.

[10] F. Bayart and E. Matheron, How to get common universal vectors, Indiana Univ. Math. J. 56 (2007), 553-580.

[11] —, 一, Dynamics of Linear Operators, Cambridge Tracts in Math. 179, Cambridge Univ. Press, 2009.

[12] L. Bernal-González, Common hypercyclic functions for multiples of convolution and non-convolution operators, Proc. Amer. Math. Soc. 137 (2009), 3787-3795.

[13] J. Bonet, F. Martínez-Giménez and A. Peris, Linear chaos on Fréchet spaces, in: Dynamical Systems and Functional Equations (Murcia, 2000), Int. J. Bifur. Chaos Appl. Sci. Engrg. 13 (2003), 1649-1655.

[14] P. S. Bourdon and N. S. Feldman, Somewhere dense orbits are everywhere dense, Indiana Univ. Math. J. 52 (2003), 811-819.

[15] K. C. Chan and R. Sanders, Common supercyclic vectors for a path of operators, J. Math. Anal. Appl. 337 (2008), 646-658.

[16] - - - Two criteria for a path of operators to have common hypercyclic vectors, J. Operator Theory 61 (2009), 191-223.

[17] - , - An SOT-dense path of chaotic operators with common hypercyclic vectors, ibid., to appear.

[18] G. Costakis, On a conjecture of D. Herrero concerning hypercyclic operators, C. R. Acad. Sci. Paris 330 (2000), 179-182.

[19] —, Approximation by translates of entire functions, in: Complex and Harmonic Analysis, DEStech Publ., Lancaster, PA, 2007, 213-219.

[20] G. Costakis and D. Hadjiloucas, Somewhere dense Cesàro orbits and rotations of Cesàro hypercyclic operators, Studia Math. 175 (2006), 249-269.

[21] G. Costakis and P. Mavroudis, Common hypercyclic entire functions for multiples of differential operators, Colloq. Math. 111 (2008), 199-203.

[22] G. Costakis and M. Sambarino, Genericity of wild holomorphic functions and common hypercyclic vectors, Adv. Math. 182 (2004), 278-306.

[23] R. Ellis, Lectures on Topological Dynamics, W. A. Benjamin, New York, 1969.

[24] N. S. Feldman, (A survey of) the dynamics of cohyponormal operators, in: Contemp. Math. 321, Amer. Math. Soc., 2003, 71-85.

[25] E. A. Gallardo-Gutiérrez and J. R. Partington, Common hypercyclic vectors for families of operators, Proc. Amer. Math. Soc. 136 (2008), 119-126.

[26] K.-G. Grosse-Erdmann, Universal families and hypercyclic operators, Bull. Amer. Math. Soc. 36 (1999), 345-381. 
[27] K.-G. Grosse-Erdmann, Recent developments in hypercyclicity, RACSAM Rev. R. Acad. Cienc. Exactas Fis. Nat. Ser. A Mat. (2003), 273-286.

[28] -, Dynamics of linear operators, in: Topics in Complex Analysis and Operator Theory, Univ. Malaga, Malaga, 2007, 41-84.

[29] K.-G. Grosse-Erdmann, F. León-Saavedra and A. Piqueras-Lerena, The iterates of a map with dense orbit, Acta Sci. Math. (Szeged) 74 (2008), 245-257.

[30] K.-G. Grosse-Erdmann and A. Peris, Linear Chaos, Universitext, Springer, to appear.

[31] G. H. Hardy and E. M. Wright, An Introduction to the Theory of Numbers, 5th ed., Oxford Univ. Press, 1979.

[32] D. A. Herrero, Limits of hypercyclic and supercyclic operators, J. Funct. Anal. 99 (1991), 179-190.

[33] F. León-Saavedra, Operators with hypercyclic Cesàro means, Studia Math. 152 (2002), 201-215.

[34] F. León-Saavedra and V. Müller, Rotation of hypercyclic and supercyclic operators, Integral Equations Operator Theory 50 (2004), 385-391.

[35] F. León-Saavedra, A. Piqueras-Lerena and J. B. Seoane-Sepúlveda, Orbits of Cesàro type operators, Math. Nachr. 282 (2009), 764-773.

[36] M. Marano and H. N. Salas, Ansari's theorem revisited and the infinite torus, Bull. Belg. Math. Soc. Simon Stevin 16 (2009), 481-492.

[37] A. Montes-Rodríguez and H. N. Salas, Supercyclic subspaces, Bull. London Math. Soc. 35 (2003), 721-737.

[38] A. Peris, Multi-hypercyclic operators are hypercyclic, Math. Z. 236 (2001), 778-786.

[39] W. Rudin, Real and Complex Analysis, McGraw-Hill, 1966.

[40] H. N. Salas, Supercyclicity and weighted shifts, Studia Math. 135 (1999), 55-74.

[41] R. Sanders, Common hypercyclic vectors and the hypercyclicity criterion, Integral Equations Operator Theory 65 (2009), 131-149.

[42] S. Shkarin, Universal elements for non-linear operators and their applications, J. Math. Anal. Appl. 348 (2008), 193-210.

[43] -, Remarks on common hypercyclic vectors, J. Funct. Anal. 258 (2010), 132-160.

[44] J. Wengenroth, Hypercyclic operators on non-locally convex spaces, Proc. Amer. Math. Soc. 131 (2003), 1759-1761.

George Costakis

Department of Mathematics

University of Crete

Knossos Avenue

GR-71409 Heraklion, Crete, Greece

E-mail: costakis@math.uoc.gr

Received September 22, 2009

Revised version November 12, 2010

(6699) 